\title{
Airway management changes in patients with mucopolysaccharidoses: the role of video laryngoscopy
}

\author{
Brittney M. Clark, MD · Juraj Sprung, MD, PhD • Toby N. Weingarten, MD • \\ Mary E. Warner, MD
}

Received: 15 May 2017/ Accepted: 26 May 2017/Published online: 7 June 2017

(c) Canadian Anesthesiologists' Society 2017

\section{To the Editor,}

Mucopolysaccharidoses (MPS) are lysosomal storage diseases resulting in pathological glycosaminoglycan accumulations. ${ }^{1}$ Craniofacial and skeletal abnormalities in these patients include macroglossia, tonsil and adenoid hypertrophy, odontoid hypoplasia, and atlantoaxial instability. $^{2-4}$ Because these anomalies can complicate airway management, intubation with flexible bronchoscopy (FB) has traditionally been used in our practice in selected MPS patients. In 2009, video laryngoscopy (VL) was introduced to our practice. Here, we describe the airway management of MPS patients who underwent general anesthesia from 2000 to 2015 , before and after the introduction of VL. This retrospective study was approved by our institutional review board (No. 15004737; approved on July 7, 2015).

To determine whether VL changed clinical practice, the study was divided into two periods - before and after the introduction of VL. Altogether, 18 MPS patients underwent 49 procedures during the study period. The Table (available as Electronic Supplementary Material [ESM]) summarizes the patients' characteristics. Seven patients presented with pre-established airways (five with oral endotracheal tubes [ETTs] and two with tracheostomies). Six others were managed with mask ventilation, which was difficult in two of the six (patients

Electronic supplementary material The online version of this article (doi:10.1007/s12630-017-0906-0) contains supplementary material, which is available to authorized users.

B. M. Clark, MD · J. Sprung, MD, PhD ( $₫)$.

T. N. Weingarten, MD - M. E. Warner, MD

Department of Anesthesiology and Perioperative Medicine,

Mayo Clinic, Rochester, MN, USA

e-mail: sprung.juraj@mayo.edu
15 and 16, both with type IV MPS). For 15 procedures, tracheal intubation was electively secured with either FB intubation (patients $3,10,13,16,17$ ) or VL (patients 7 [×2], $9[\times 3], 11,12,13[\times 2], 18)$. VL was used for rescue in two additional cases (patients 6 and 15, described below) (see ESM for specific patient characteristics). Airway management was uneventful during 19 procedures (17 direct laryngoscopies [DL] and establishment of two laryngeal mask supraglottic airways [LM]).

Initial airway management attempts failed in three patients. Patients 3 had 5 uderwet unsuccessful FB attempts by one anesthesiologist, with eventual successful FB conducted by a bronchoscopy consultant. Patient 6 had two failed DLs followed by successful VL. Patient 15 had three failed LMs followed by VL-assisted FB (VL alone obtained only a partial view of the epiglottis). The Figure (and ESM video) show how VL visualization could facilitate FB advancement.

A comparison of the pre-VL and post-VL periods revealed that during the pre-VL period 12 cases were managed with traditional techniques (11 DL, one LM) and five with FB. During the post-VL period, six cases were managed with DL and 12 with VL (ten elective and two rescues [DL and LM failures]), suggesting that the introduction of $\mathrm{VL}$ affected airway management in these patients $(P=0.044)$.

Our main finding was that, following its introduction, VL has become the preferred intubation tool for MPS patients. Overall, 17/36 (47\%) native airways were secured with advanced techniques, with 12 of 18 cases during the contemporary period managed with VL. VL is imperfect, however, as illustrated by patient 15 , in whom VL could not obtain a glottic view. This example provides a cautionary reminder that emerging technologies can still fail, so backup plans must be available. In that particular 


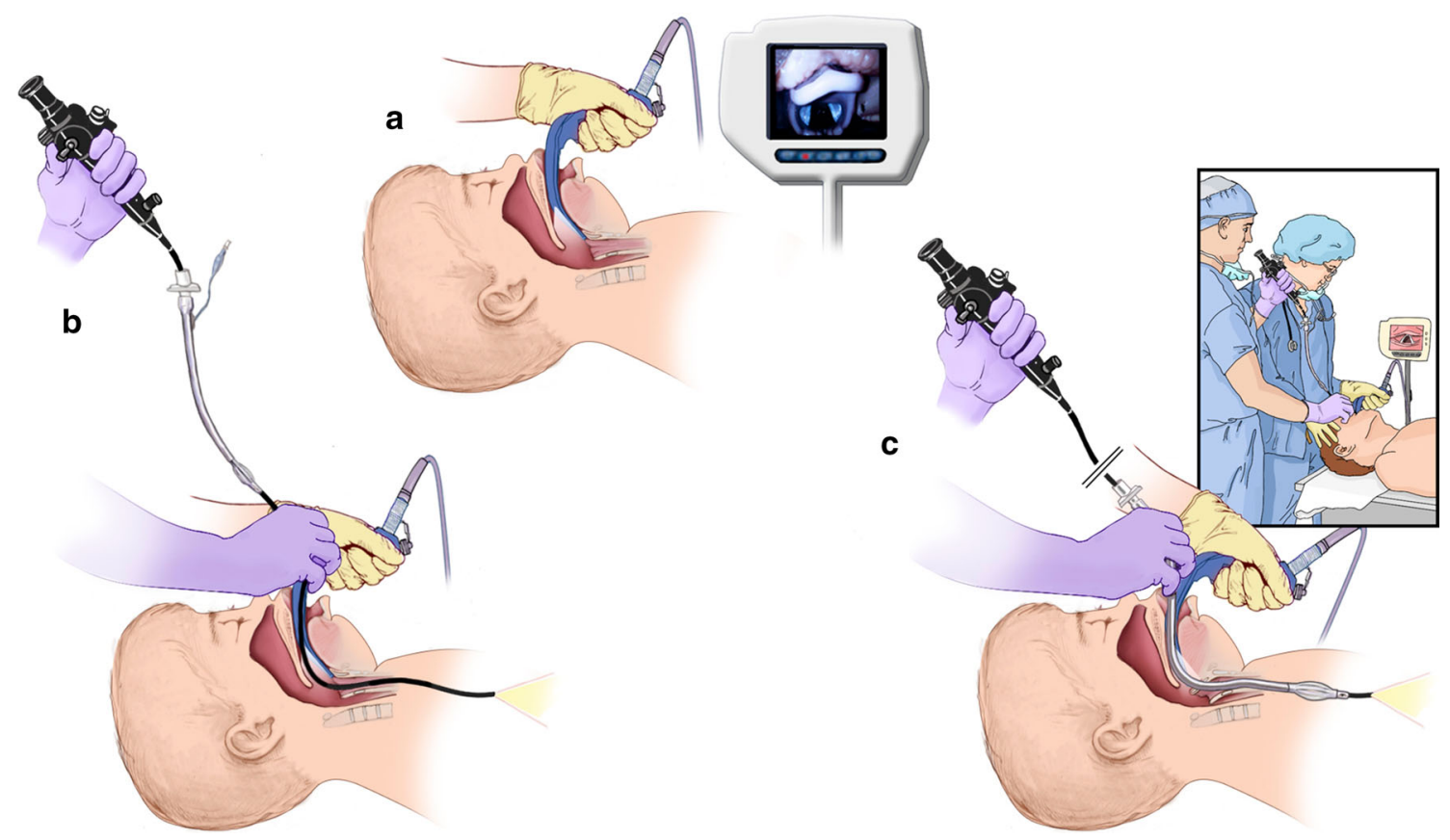

Figure A technique for endotracheal intubation that involves two providers using video laryngoscopy (VL) and flexible bronchoscopy (FB). The first anesthesiologist visualizes the glottic opening with VL and displays it on the monitor (A). A second anesthesiologist,

case, the airway was secured with the simultaneous use of VL and FB (where the first provider used VL to obtain the best achievable glottic view (Figure A), followed by the second provider using the VL monitor to guide an FB preloaded with an ETT into the trachea (Figure B). Use of the FB as a malleable, maneuverable ETT bougie facilitates entering a significantly distorted glottis. It also extends the visualization field from the FB eyepiece if the glottis cannot be visualized with VL. Once the FB is positioned in the distal trachea, the ETT is advanced. The $\mathrm{VL}$ remains in place to allow retraction of redundant tissues and visually confirms successful ETT intubation (Figure C).

Thus, VL has become the preferred tool in our practice to manage airways in MPS patients undergoing general anesthesia. VL is not failsafe, however, and anesthesiologists must have in place an alternative plan for airway management. watching the monitor, then inserts the conventional FB preloaded with an endotracheal tube (ETT) (B) into the trachea (C). After FB insertion, the ETT is advanced into the trachea over the FB (used with permission of Mayo Foundation for Medical Education and Research)

Conflicts of interest None declared.

Editor Responsiblity This submission was handled by Dr. Hilary P. Grocott, Editor-in-Chief, Canadian Journal of Anesthesia.

\section{References}

1. Muenzer J. The mucopolysaccharidoses: a heterogeneous group of disorders with variable pediatric presentations. J Pediatr 2004; 144(5 Suppl): S27-34.

2. Walker R, Belani $K G$, Braunlin EA, et al. Anaesthesia and airway management in mucopolysaccharidosis. J Inherit Metab Dis 2013; 36: 211-9.

3. Arn P, Bruce IA, Wraith JE, Travers H, Fallet $S$. Airway-related symptoms and surgeries in patients with mucopolysaccharidosis I. Ann Otol Rhinol Laryngol 2015; 124: 198-205.

4. Gurumurthy T, Shailaja S, Kishan S, Stephen M. Management of an anticipated difficult airway in Hurler's syndrome. J Anaesthesiol Clin Pharmacol 2014; 30: 558-61. 